\title{
Contamination of RR Lyrae stars from Binary Evolution Pulsators
}

\author{
P. Karczmarek* \\ Warsaw University Observatory, Al. Ujazdowskie 4, 00-478, Warsaw, Poland
}

\begin{abstract}
A Binary Evolution Pulsator (BEP) is a low-mass $\left(0.26 \mathfrak{M}_{\odot}\right)$ member of a binary system, which pulsates as a result of a former mass transfer to its companion. The BEP mimics RR Lyrae-type pulsations, but has completely different internal structure and evolution history. Although there is only one known BEP (OGLE-BLG-RRLYR02792), it has been estimated that approximately $0.2 \%$ of objects classified as RR Lyrae stars can be undetected Binary Evolution Pulsators. In the present work, this contamination value is re-evaluated using the population synthesis method. The output falls inside a range of values dependent on tuning the parameters in the StarTrack code, and varies from $0.06 \%$ to $0.43 \%$.
\end{abstract}

Key words: methods: numerical - variables: RR Lyrae - binaries

\section{INTRODUCTION}

RR Lyrae (RRL) stars reside in a small area of the instability strip (IS) in the Hertzsprung-Russel diagram, limited by effective temperature and luminosity given below [2]:

$$
\begin{array}{r}
5000 \mathrm{~K}<T_{\text {eff, RR }}<7400 \mathrm{~K}, \\
16 \mathfrak{L}_{\odot}<L_{\mathrm{RR}}<100 \mathfrak{L}_{\odot} .
\end{array}
$$

Stellar evolution models find RRL mass in a narrow range $0.6-0.8 \mathfrak{M}_{\odot}$, which corresponds to RRL age of $10-12 \mathrm{Gyr}$. These properties make RR Lyrae pulsators precise tools for determining the age and distance to the hosting galaxies and clusters.

With a growing number of pulsating variables found in binary systems, it is surprising that no RR Lyrae binary has been reported so far. The discovery of a RR Lyr-type pulsator in a binary system OGLE-BLG-RRLYR-02792 [6] was even more surprising, as the pulsator's mass was only $0.26 \mathfrak{M}_{\odot}$ - clearly too small for a bona fide RR Lyrae star. Thus, a RRL-type pulsator is assumed to be the product of a mass transfer (MT) episode, during which the initially more massive component removed most of its envelope and exposed the degenerated helium core, placing itself in a narrow range of effective temperatures and luminosities characteristic for RR Lyrae IS. The mass transfer was the key stage in the binary evolution, producing a pulsator with such a small mass within the Hubble time. Because of its peculiar evolution, the pulsator earned the name BEP (Binary Evolution Pulsator).

Preliminarily estimations suggest that approximately $0.2 \%$ of known RR Lyrae stars are actu-

\footnotetext{
* pkarczmarek@astrouw . edu.pl
}

(C) P. Karczmarek, 2015 ally BEPs [6] which cannot be distinguished from bona fide RRLs due to the lack of binarity indications, e.g. eclipses. This contamination value implies that BEPs should be scarce enough to not affect any statistics-based calculations involving RR Lyrae stars, like age or distance determinations. To gain confidence, the contamination value is revised with use of a synthesis population code StarTrack [1]. The code, equipped with up-to-date stellar formulae governing both single and binary evolution, proceeds the evolution of a large number of binaries (one at a time), and deliver statistical occurrence of objects of interest (with predetermined set of parameters) in comparison with the total sample. First results from StarTrack, showing various evolutionary tracks for simulated BEPs, were introduced by Karczmarek in 2012 [4]. In this work, the BEP contamination value is presented as strongly dependent on the parameters governing MT in the StarTrack code. The discussion of this result points the direction of further study devoted to BEP.

\section{StARTRACK CALCULATIONS}

The percentage of contamination of RRL stars from BEPs is given by:

$$
C=\frac{N_{\mathrm{BEP}}}{N_{\mathrm{RRL}}} \times 100 \%,
$$

where $N_{\mathrm{BEP}}$ and $N_{\mathrm{RRL}}$ are the number of BEP and RR Lyrae stars found in a given sample, respectively. For this study, the size of the sample was $10^{5}$ objects within a wide mass range $0.3-150.0 \mathfrak{M}_{\odot}$, and the probability of drawing the star of a mass $m$ was given 
by the Initial Mass Function (IMF) [5]:

$$
\xi(m)= \begin{cases}0 & \text { if } m<0.1 \mathfrak{M}_{\odot}, \\ 0.29056 m^{-1.3} & \text { if } 0.1 \mathfrak{M}_{\odot} \leq m<0.5 \mathfrak{M}_{\odot}, \\ 0.15571 m^{-2.2} & \text { if } 0.5 \mathfrak{M}_{\odot} \leq m<1.0 \mathfrak{M}_{\odot}, \\ 0.15571 m^{-2.7} & \text { if } m \geq 1.0 \mathfrak{M}_{\odot}\end{cases}
$$

As Pietrzyński stated, $20 \%$ of stars with initial masses in range $0.8-0.9 \mathfrak{M} \odot$ can become RR Lyrae stars [6]. In this work, the number of RR Lyrae stars, $N_{\text {RRL }}$, was found by filtering from a total sample only $20 \%$ of objects with initial masses in a range $0.8-0.9 \mathfrak{M}_{\odot}$.

The procedure of choosing BEP from a given sample was more complicated, because it accounted for not one, but four parameters: initial mass of primary component $m$ (in units of $\mathfrak{M}_{\odot}$ ), initial mass ratio $q$, initial eccentricity $e$, and initial separation $a$ (in units of $\left.\mathfrak{R}_{\odot}\right)^{1}$. Noteworthy, $50 \%$ of all systems are considered to be binary or multiple systems. Because BEP is always a product of binary evolution, the probability of its occurrence needs to be reduced by $50 \%$, adequately to the occurrence of binary systems among all systems. Finally, noting that BEP crosses the Instability Strip (IS) a hundred times faster than RR Lyrae star, one should expect to detect one hundred times fewer BEP events, than if the IS crossing times for BEP and RRL were similar. Above considerations are summarized in the following formula:

$$
N_{\mathrm{BEP}}=S T(m, q, e, a) \times 0.5 \times 0.01,
$$

where $S T(m, q, e, a)$ represents the StarTrack output, i. e. the number of BEPs found in the sample of $10^{5}$ binaries.

A simple filter was implemented to StarTrack code in order to find BEPs in the total sample. The BEP object was detected if at any stage of binary evolution all of the following conditions were simultaneously satisfied: (i) the mass of an object was less than $0.45 \mathfrak{M}_{\odot}$, (ii) the object was evolved at least to Red Giant stage, (iii) the effective temperature and the luminosity were within the range of values given by Eq. (1), and (iv) the MT was not ongoing. Figure 1 shows a mesh of BEPs occurrence as a function of the initial mass of more massive component $A$, $M_{0, \mathrm{~A}}$, and mass ratio $q=M_{0, \mathrm{~A}} / M_{0, \mathrm{~B}}$ for different initial periods from 2 to 20 days. The eccentricity was assumed zero at all times (this was justified, because the system needs to circularize its orbit prior to the onset of MT).

Mass transfer can proceed in general in two forms: stable MT in the form of the Roche Lobe Over Flow (RLOF) or unstable MT in the form of an instant phase of Common Envelope (CE). The BEP favours the stable MT scenario, i.e. the simulations fail to recover the BEP system assuming CE scenario. Thus, the algorithm governing MT in the StarTrack code was tuned to choose RLOF over CE scenario whenever the status of MT was unclear. The output mesh of BEP occurrence produced by the new version of StarTrack code is shown in Figure 2. The comparison of both figures indicates that more BEPs with larger mass ratio (up to $q=3$ ) are produced when "stable MT" option is on.

Finally, the synthesis population was run for both versions of the StarTrack code. Each time a BEP was found in the binary, the parameters of this system were saved, and the total sum of BEPs in the sample was increased by one. In this way, two different databases were created and two different contamination numbers were derived - for the original version and for the "stable MT" version of the ST code. The results, especially the contamination number, are presented in the next section.

\section{RESULTS AND CONCLUSIONS}

The contamination ratio of RRL stars from BEPs was calculated from Eq. (2). The number of RRLs and the number of BEPs were found independently in a total sample of $10^{5}$ elements, in a manner described in a previous section. In the case of BEP, the population synthesis was performed for two scenarios of mass transfer (original MT versus "stable MT" version). The contamination ratio $C$ is $0.06 \%$ in the case of the original treating of MT, and $0.43 \%$ in the case of enhanced stability of MT. These values define the range of the contamination ratio, and Pietrzyński's value $0.2 \%$ [6] lies well within this range. It should be noted that the choice of eccentricity distribution [3] does not affect neither the number of encountered BEPs nor the characteristics of found BEPs.

A more careful investigation of selected BEP properties is presented in Figures 3, 4, and 5. General conclusions drawn from these histograms are as follows. The initial mass of BEP progenitor can be as high as $4.5 \mathfrak{M}_{\odot}$ for both versions of MT treatment and for both eccentricity distributions, although the most preferred mass range for BEP occurrence is approximately $1.7 \mathfrak{M}_{\odot}$ and $1.0 \mathfrak{M}_{\odot}$ for original and "stable MT" versions, respectively. BEP masses cluster around values $0.26-0.27 \mathfrak{M}_{\odot}$, but more massive cases are also visible - they have higher luminosities (close to the upper limit from Eq. (1)) and reside in binaries with wider orbits. Indeed, Figure 5 shows two distinct peaks for BEP orbital period distributions, with the smaller peak corresponding to more massive and more luminous BEPs. This suggest that BEPs may contaminate not only RR Lyrae stars but

\footnotetext{
${ }^{1}$ Initial parameters were drawn from probability distributions as follows: (i) $m$ was drawn from the IMF as given in Eq. (3) over the same mass range $[0.3,150.0]$, (ii) $q$ was drawn from uniform distribution over a range $[0,1]$, (iii) $e$ was drown from two different probability distributions: $f_{1}(e)=2 e, f_{2}(e)=\mathcal{N}\left(0.26,0.14^{2}\right)$ [3]; the comparison of outputs for these two distributions is presented in the next section, (vi) $a$ was drawn from uniform distribution of $\log _{10}(a)$ over a range $\left[a_{\min }, 10^{5}\right]$; $a_{\text {min }}$ denotes the smallest separation without initial merging of components. See [1] for details.
} 

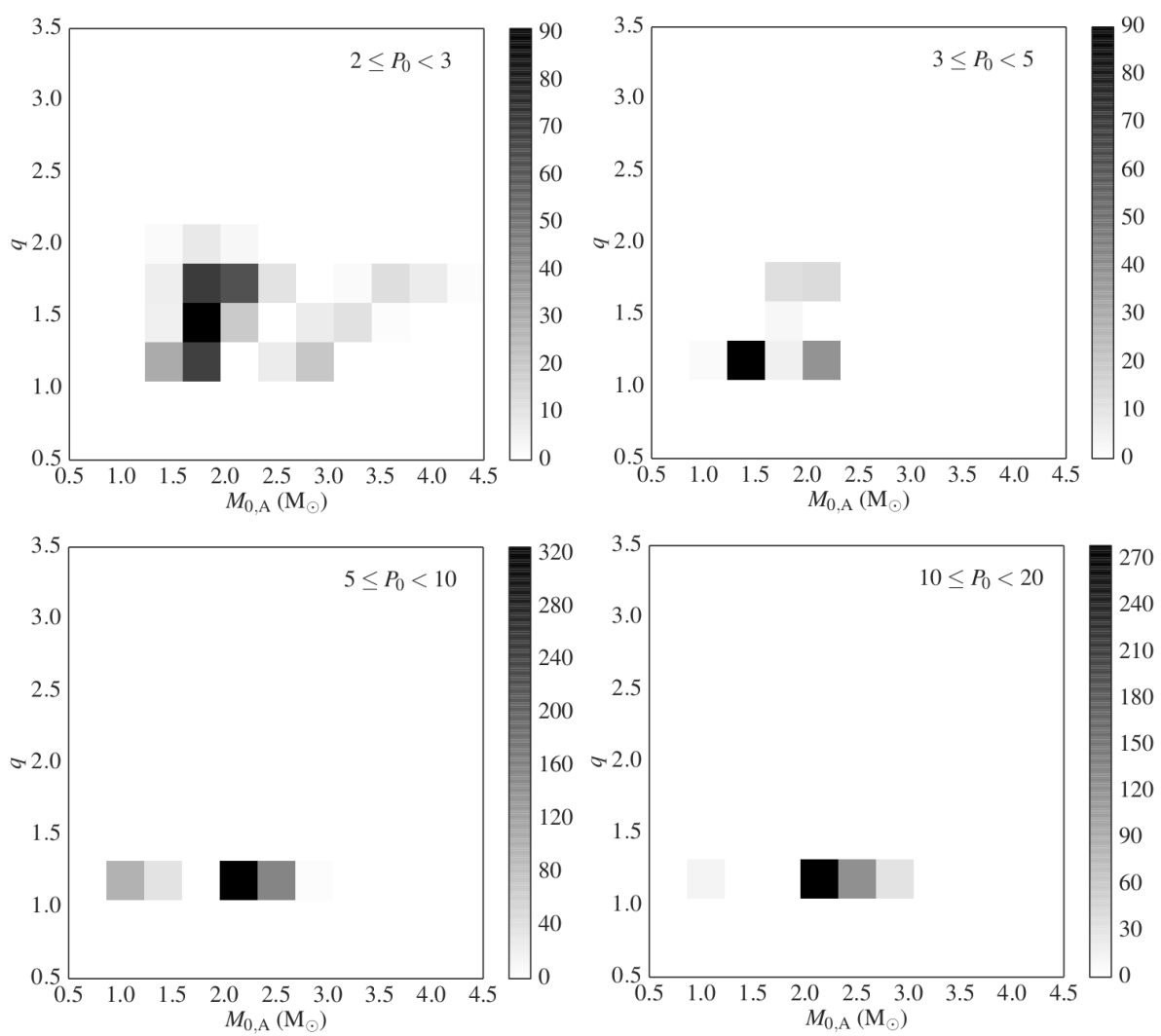

Fig. 1: The mesh of occurance of Binary Evolution Pulsator (BEP) depending on the initial mass of more massive component $A, M_{0, \mathrm{~A}}$, and mass ratio $q=M_{0, \mathrm{~A}} / M_{0, \mathrm{~B}}$ of components in a binary system for four different ranges of initial periods, from $2 \mathrm{~d}$ to $20 \mathrm{~d}$, and assuming circular orbits $(e=0)$. If at any time of a system's evolution, the properties of a component matched the BEP characteristics (see text for details), this component was considered as BEP. This simulation used the StarTrack code with the original treatment of mass transfer.

also other pulsating variables up in the Instability Strip, like type II Cepheids, but the evaluation of such statement is beyond the scope of this paper.

The obvious and the most important uncertainty of the contamination ratio $C$ is the treating of mass transfer. Further evaluation of the mass transfer phase is crucial to obtain the precise value of contamination ratio. In this work, the range of contamination values was established for two different methods of MT treatment, yielding $C \in[0.06 \%, 0.43 \%]$. The upper limit $0.43 \%$ is still negligible in the analysis of large sample RR Lyrae stars, e.g. for determining clusters' age or distance, as predicted earlier [6]. Nevertheless, the presence of objects with such peculiar properties points an interesting direction of further studying for pulsation theory [7].

\section{ACKNOWLEDGEMENT}

It is a pleasure to thank G. Pietrzyński for substantive guidance to this project. I am gratefull to
K. Belczynski for sharing the StarTrack code and for many useful and valuable instructions. This research is supported by the Polish National Science Centre grant PRELUDIUM 2012/07/N/ST9/04246.

\section{REFERENCES}

[1] Belczynski K., Kalogera V., Rasio F. A. et al. 2008, ApJS, 174, 223

[2] Bono G., Caputo F., Cassisi S., Incerpi R. \& Marconi M. 1997, AJ, 483, 811

[3] Duquennoy A. \& Mayor M. 1991, A\&A, 248, 485

[4] Karczmarek P. 2012, Advances in Astronomy and Space Physics, 2, 135

[5] Kroupa P., Tout C. A. \& Gilmore G. 1993, MNRAS, 262, 545

[6] Pietrzyński G., Thompson I. B., Gieren W. et al. 2012, Nature, 484, 75

[7] Smolec R., Pietrzyński G., Graczyk D. et al. 2013, MNRAS, 428, 3034 

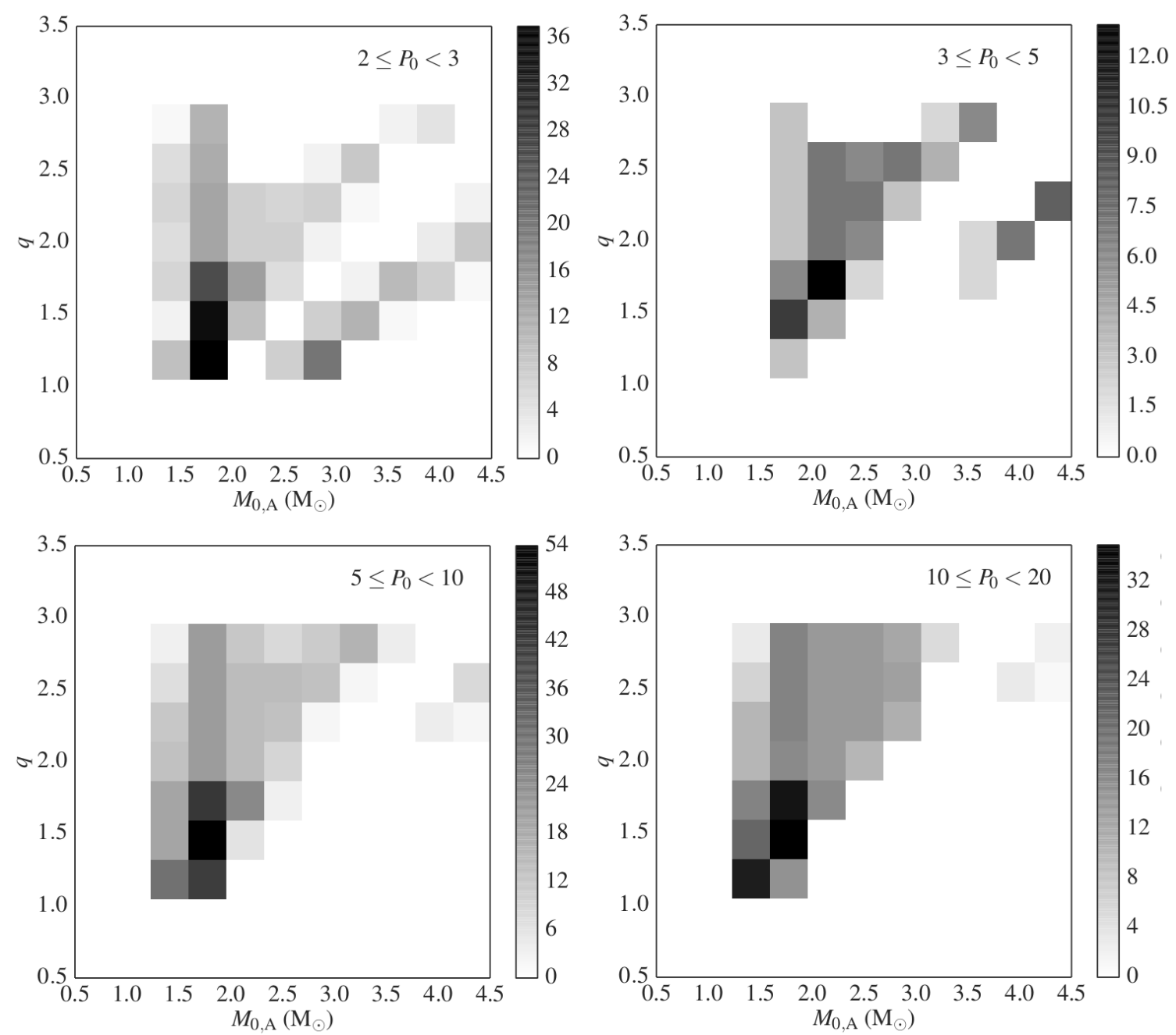

Fig. 2: The mesh of occurance of Binary Evolution Pulsator (BEP) depending on the initial mass of more massive component $A, M_{0, \mathrm{~A}}$, and mass ratio $q=M_{0, \mathrm{~A}} / M_{0, \mathrm{~B}}$ of components in a binary system for four different ranges of initial periods, from $2 \mathrm{~d}$ to $20 \mathrm{~d}$, and assuming circular orbits $(e=0)$. If at any time of a system's evolution, the properties of a component matched the BEP characteristics (see text for details), this component was considered as BEP. This simulation used the StarTrack code with the enhanced stability of mass transfer. 

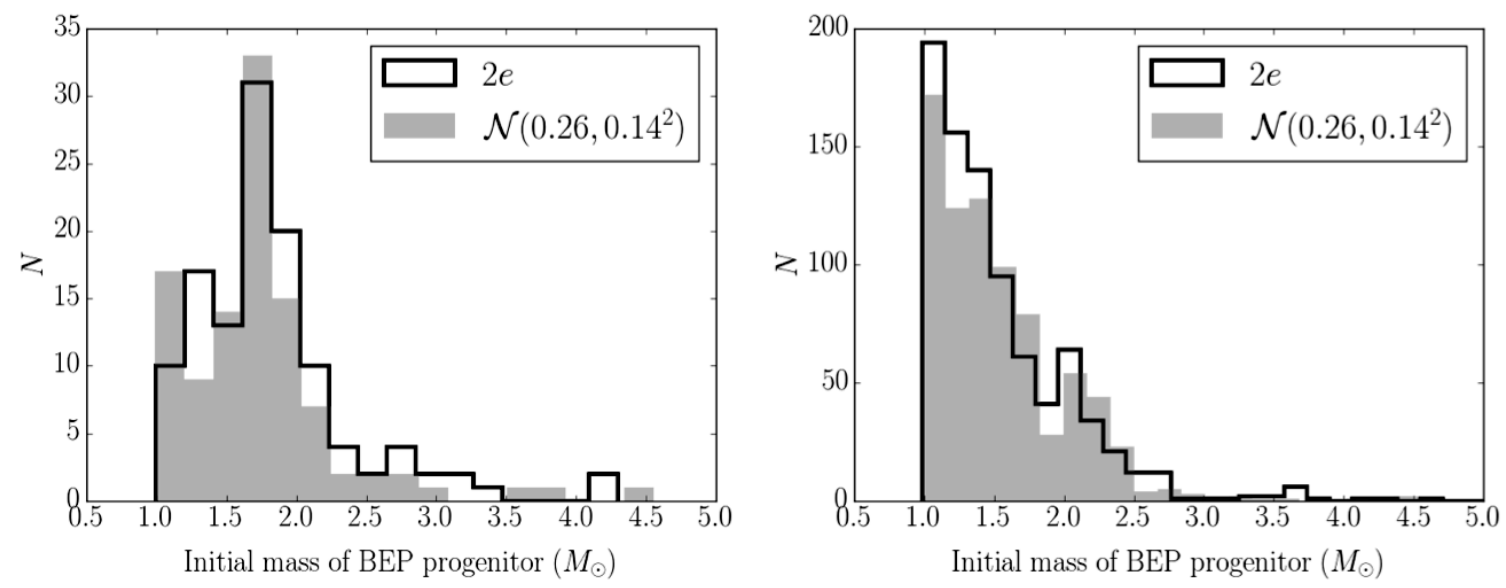

Fig. 3: Distributions of the initial mass of BEP progenitor for two initial eccentricity functions; left: original MT, right: "stable MT".
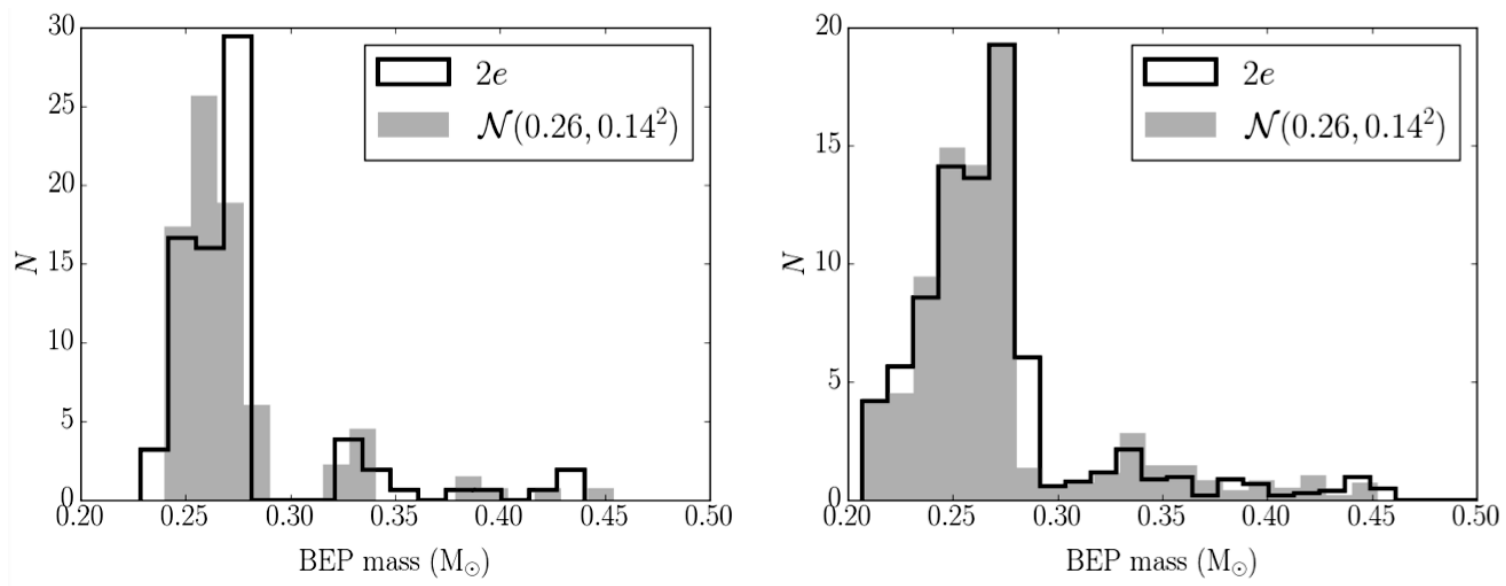

Fig. 4: Distributions of the BEP mass for two initial eccentricity functions; left: original MT, right: "stable MT".
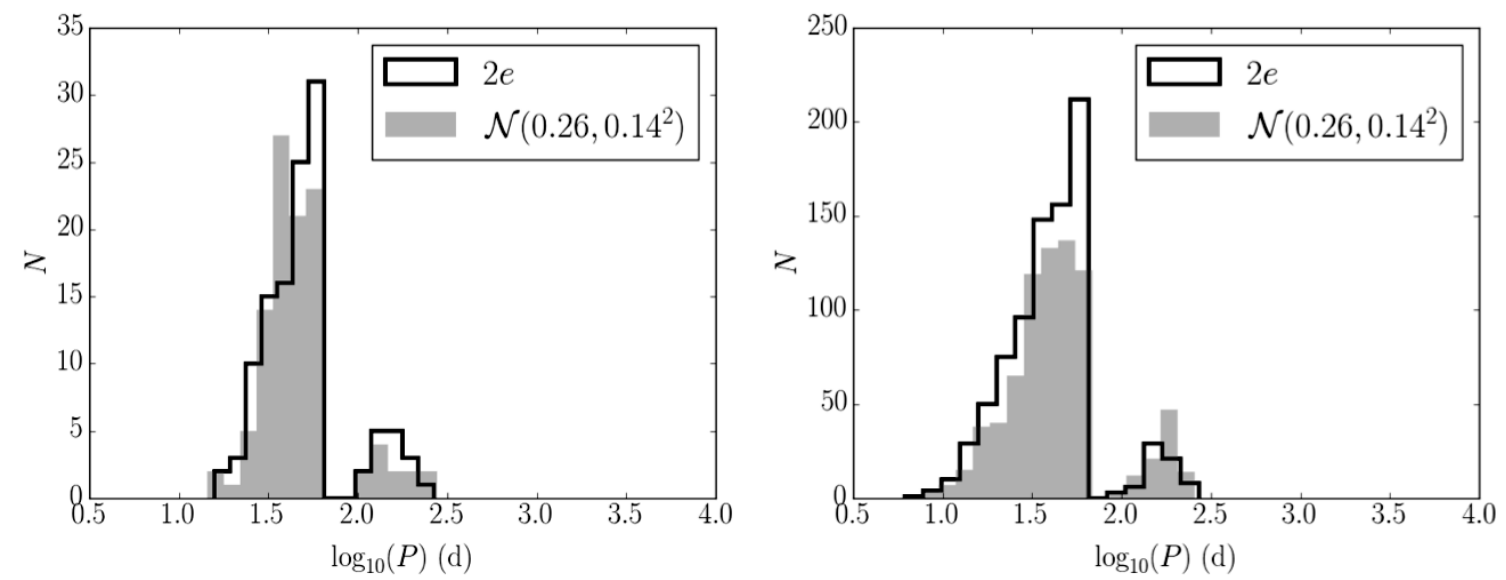

Fig. 5: Distributions of the orbital period of a binary with BEP component for two initial eccentricity functions; left: original MT, right: "stable MT". 